\title{
ON THE SET OF PERIODS OF THE GRAPH HOMEOMORPHISMS
}

\author{
MÁRCIO R.A. GOUVEIA ${ }^{1,2}$ AND JAUME LLIBRE ${ }^{1}$
}

\begin{abstract}
In this paper we characterize all possible sets of periods of homeomorphisms defined on some classes of finite connected compact graphs.
\end{abstract}

\section{INTRODUCTION}

Here a (topological graph) or simply a graph $G$ is a compact set formed by a finite union of vertices (points) and edges, which are homeomorphic to a non-empty open interval of the real line, and are pairwise disjoint. The boundary of one edge is formed either by two vertices, or by a unique vertex. Moreover, the graphs that we consider here always are connected.

We identify a circle with the unit circle $\mathbb{S}^{1}$ centered at the origin of the complex plane. A circuit (or loop) of a graph $G$ is any subset of $G$ homeomorphic to $\mathbb{S}^{1}$. A tree is a graph without circuits. The set of vertices of a graph $G$ will be denoted by $V(G)$. Clearly $V(G)$ is finite.

Let $G$ be a graph and $z \in G$. Then, we consider a small open neighborhood $U$ (in $G$ ) of $z$ such that $\mathrm{Cl}(U)$ is a tree. The number of connected components of $U \backslash\{z\}$ is called the valence of $z$ and is denoted by $\operatorname{Val}(z)$. Observe that this definition is independent of the choice of $U$ if it is sufficiently small, and that $\operatorname{Val}(z) \neq 2$ implies that $z \in V(G)$. A vertex of valence 1 is called an endpoint of $G$ and a vertex of valence larger than 2 is called a branching point of $G$.

Let $f: G \rightarrow G$ be a continuous map. A point $z \in G$ such that $f(z)=z$ is called a fixed point or a periodic point of period 1. The point $z \in G$ is periodic of period $m>1$ if $f^{m}(z)=z$ and $f^{k}(z) \neq z$ for $k=1, \ldots, m-1$. Of course, in the whole paper $f^{m}(z)$ denotes the $m$-th iterate of the point $z$ by the map $f$. We denote by $\operatorname{Per}(f)$ the set of periods of all periodic points of $f$.

In this work our aim is to characterize the sets $\operatorname{Per}(f)$ when $f: G \rightarrow G$ is a homeomorphism of a given graph $G$. As we will see this objective is only reached for some classes of graphs, the full characterization for every graph looks as a very hard problem.

2010 Mathematics Subject Classification. 37E25, 37B40.

Key words and phrases. homeomorphisms, topological graph, periods, periodic points. 


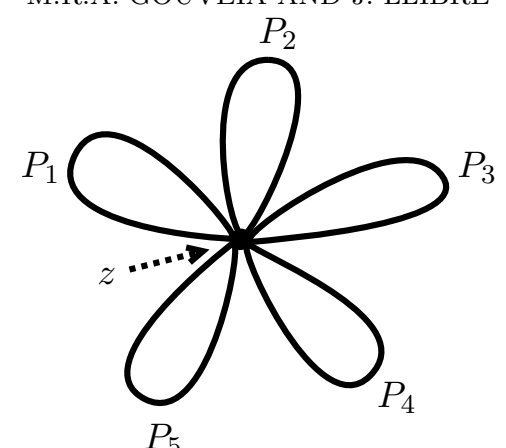

Figure 1. A 5-flower graph.

Probably the first result on the set of periods of a homeomorphism of a graph is the following one due to Fuller [3]. See section 2 for the definition of independent oriented loops.

Theorem 1. Let $G$ be a graph with $c$ independent oriented loops and let $f: G \rightarrow G$ be a homeomorphism. Then, the following statements hold.

(a) If $c=0$ (i.e. $G$ is a tree), then $1 \in \operatorname{Per}(f)$.

(b) If $c>1$, then $\operatorname{Per}(f) \cap\{1,2, \ldots, c\} \neq \emptyset$.

In fact Fuller does not provide Theorem 1, he provided a more general result that restricted to graphs becomes Theorem 1, see for details section 2.

The characterizations of the sets of periods for the homeomorphisms on a closed interval $I$ or on the circle $\mathbb{S}^{1}$ are well known for the mathematicians working in topological dynamics, see the next two theorems, but since it is not easy to find their proofs in the literature we provide a proof of these two theorems in section 3 .

Theorem 2 (Interval Theorem). Let I be a non-degenerate closed interval (i.e. different from a point), and let $f: I \rightarrow I$ be a homeomorphism. Then

$$
\operatorname{Per}(f)= \begin{cases}\{1\} & \text { if } f \text { is increasing, } \\ \{1,2\} & \text { if } f \text { is decreasing. }\end{cases}
$$

As usual $\mathbb{Q}$ and $\mathbb{R}$ denote the sets of rational and real numbers respectively. See the definition of rotation number $\rho(f) \in \mathbb{R}$ for a homeomorphism $f$ : $\mathbb{S}^{1} \rightarrow \mathbb{S}^{1}$ which preserves the orientation in section 3 .

Theorem 3 (Circle Theorem). Let $f: \mathbb{S}^{1} \rightarrow \mathbb{S}^{1}$ be a homeomorphism.

(a) If $f$ preserves the orientation, then

$$
\operatorname{Per}(f)=\left\{\begin{array}{cl}
\emptyset & \text { if } \rho(f) \notin \mathbb{Q}, \\
\{n\} & \text { if } \rho(f)=\frac{k}{n} \text { with } \operatorname{gcd}(k, n)=1 .
\end{array}\right.
$$

(b) If $f$ reverses the orientation, then $\operatorname{Per}(f)$ is either $\{1\}$, or $\{1,2\}$. 


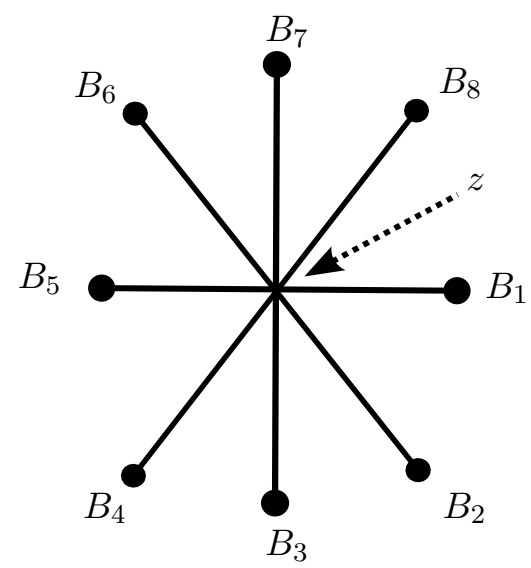

Figure 2. The 8-odd graph.

A $p$-flower graph is a graph with a unique branching point $z$ and $p>1$ edges all having a unique endpoint, the point $z$, equal for all of them. So, this graph has $p$ independent loops, each one is called a petal. See a 5 -flower graph in Figure 1.

Theorem 4 ( $p$-Flower Theorem). Let $f: G \rightarrow G$ be a homeomorphism of a p-flower graph $G$ with $p$ petals $P_{1}, P_{2}, \ldots, P_{p}$.

(a) If $f\left(P_{l}\right)=P_{l}$ for $l=1,2, \ldots, p$, then $\operatorname{Per}(f)$ is either $\{1\}$, or $\{1,2\}$.

(b) If $f\left(P_{l}\right) \neq P_{l}$ for some $l \in\{1,2, \ldots, p\}$, then $\operatorname{Per}(f)$ is either $\{1\}$, or any subset of $\left\{1, n_{1}, n_{2}, \ldots, n_{s}, 2 n_{1}, 2 n_{2}, \ldots, 2 n_{s}\right\}$ containing the 1 , where $n_{1}, n_{2}, \ldots, n_{s}$ are arbitrary positive integers (non necessarily different) satisfying $1<n_{1}+n_{2}+\ldots+n_{s}=p$.

A graph with only one branching point $z$ with valence $b>2$ and $b$ edges having every edge the vertex $z$ and another vertex different from $z$ as endpoints always with valence 1 is called a $b$-odd graph. See an 8-odd graph in Figure 2.

Theorem 5 (b-odd Theorem). Let $f: G \rightarrow G$ be a homeomorphism of a $b$-odd graph $G$ with branching point $z$ and edges $B_{1}, B_{2}, \ldots, B_{b}$. Then the set $\operatorname{Per}(f)$ is $\{1\}$ if $f(x)=x$ for all $x \in V(G)$, or $\left\{1, n_{1}, n_{2}, \ldots, n_{s}\right\}$ otherwise, where $n_{1}, n_{2}, \ldots, n_{s}$ are positive integers (non necessarily different) satisfying $1<n_{1}+n_{2}+\ldots+n_{s}=b$.

A graph with only two vertices $z$ and $w$ and $n>1$ edges having every edge the vertices $z$ and $w$ as endpoints is called an $n$-lips graph. See a 7-lips graph in Figure 3.

Theorem 6 ( $n$-lips Theorem). Let $f: G \rightarrow G$ be a homeomorphism of the $n$-lips graph $G$ with vertices $z$ and $w$, and let $e_{1}, e_{2}, \ldots, e_{n}$ be the edges of $G$. Then the set $\operatorname{Per}(f)$ is

(a) either $\{1\}$, if $f(z)=z$ and $f\left(e_{i}\right)=e_{i}$ for all $i=1,2, \ldots, n$; 


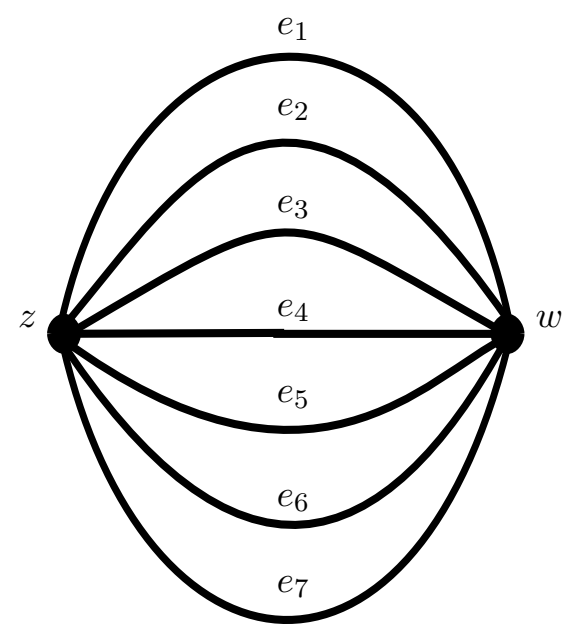

Figure 3. The 7-lips graph.

(b) or any subset of $\left\{1, n_{1}, n_{2}, \ldots, n_{s}\right\}$ including the 1 , if $f(z)=z$ and $f\left(e_{i}\right) \neq e_{i}$ for some $i \in\{1,2, \ldots, n\}$ (see the restrictions of the numbers $n_{i}$ after all the statements);

(c) or $\{1,2\}$, if $f(z) \neq z$ and $f\left(e_{i}\right)=e_{i}$ for all $i=1,2, \ldots, n$;

(d) or any subset of $\left\{2, n_{1}, n_{2}, \ldots, n_{s}, 2 n_{1}, 2 n_{2}, \ldots, 2 n_{s}\right\}$ including the set $\left\{2, n_{1}, n_{2}, \ldots, n_{s}\right\}$, if $f(z) \neq z$ and $f\left(e_{i}\right) \neq e_{i}$ for some $i \in$ $\{1,2, \ldots, n\}$,

where $n_{1}, n_{2}, \ldots, n_{s}$ are non-negative integers (non necessarily different) satisfying $1<n_{1}+n_{2}+\ldots+n_{s}=n$. The periods $2 n_{i}$ for $i=1,2, \ldots, s$ only can appear if $n_{i}$ is odd.

A graph with $p+b$ edges, where $p \geq 1$ of them are petals and the other $b \geq 1$ are not petals, having all the edges as endpoint a point $z$, is called a $(p, b)-$ graph. In this case the point $z$ has valence $2 p+b$, and it is called the main branching point of the $(p, b)$-graph. See a $(4,10)$-graph in Figure 4.

Theorem $7((p, b)$-graph Theorem). Let $f: G \rightarrow G$ be a homeomorphism of $a(p, b)$-graph $G$ with $p$ petals $P_{1}, P_{2}, \ldots, P_{p}$ and $b$ edges $B_{1}, B_{2}, \ldots$, $B_{b}$, which are not petals. Let $z$ be the main branching point of $G$. All the biggest subgraphs of $G$, which are n-lips for some $n$, are grouped as follows. Let $L_{j_{q}, 1}^{\eta_{q}}, L_{j_{q}, 2}^{\eta_{q}}, \ldots, L_{j_{q}, t_{q}}^{\eta_{q}}$ be all the $\eta_{q}$-lips subgraphs of $G$ whose two vertices are the vertex $z$ and another vertex $w_{k}$ with $k=1, \ldots, t_{q}$, each vertex $w_{k}$ has valence $\eta_{q}$, and $q=1,2, \ldots, \rho$ (see Figure 4). Then the set $\operatorname{Per}(f)$ is

(a) either $\{1\}$, or $\{1,2\}$, if $f\left(P_{l}\right)=P_{l}$ for all $l=1,2, \ldots, p$, and $f\left(B_{j}\right)=$ $B_{j}$ for all $j=1,2, \ldots, b$; 


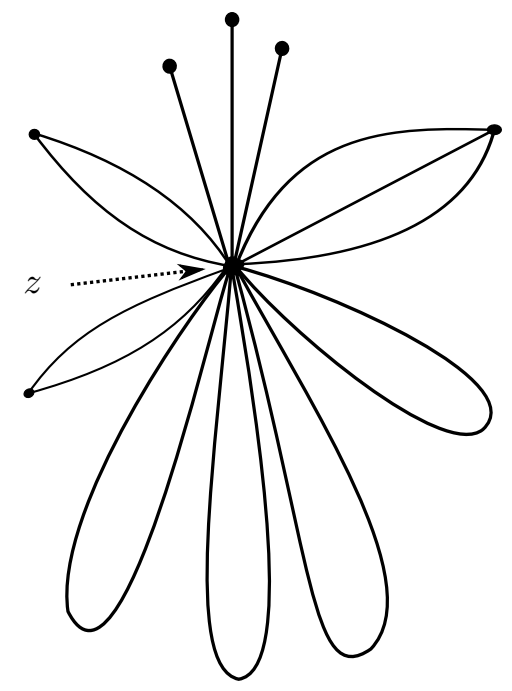

Figure 4. A $(4,10)$-graph with $\rho=q=1, \eta_{q}=3$ and $t_{q}=2$.

(b) or $\left\{1, n_{1}, n_{2}, \ldots, n_{s}\right\} \bigcup\left(\bigcup_{q=1}^{\rho}\left(\bigcup_{i=1}^{v_{q}} r_{i, q} A_{i, q}\right)\right)$,

or $\left\{1,2, n_{1}, n_{2}, \ldots, n_{s}\right\} \bigcup\left(\bigcup_{q=1}^{\rho}\left(\bigcup_{i=1}^{v_{q}} r_{i, q} A_{i, q}\right)\right)$,

if $f\left(P_{l}\right)=P_{l}$ for all $l=1,2, \ldots, p$, and $f\left(B_{j}\right) \neq B_{j}$ for some $j \in$ $\{1,2, \ldots, b\}$ (see the restrictions on the numbers $n_{i}, q, r_{i, q}$ and $v_{q}$ at the end of the statements);

(c) or $\{1\}$, or any subset of $\left\{1, k_{1}, k_{2}, \ldots, k_{u}, 2 k_{1}, 2 k_{2}, \ldots, 2 k_{u}\right\}$ containing the 1 , where $k_{1}, k_{2}, \ldots, k_{u}$ are arbitrary positive integers (non necessarily different) satisfying $1<k_{1}+k_{2}+\ldots+k_{u}=p$, if $f\left(P_{l}\right) \neq P_{l}$ for some $l \in\{1,2, \ldots, p\}$, and $f\left(B_{j}\right)=B_{j}$ for all $j=1,2, \ldots, b$;

(d) or any subset of

$\left\{1, n_{1}, \ldots, n_{s}, k_{1}, k_{2}, \ldots, k_{u}, 2 k_{1}, 2 k_{2}, \ldots, 2 k_{u}\right\} \bigcup\left(\bigcup_{q=1}^{\rho}\left(\bigcup_{i=1}^{v_{q}} r_{i, q} A_{i, q}\right)\right)$

including all the elements of this set except perhaps some of the elements of the set $\left\{k_{1}, k_{2}, \ldots, k_{u}, 2 k_{1}, 2 k_{2}, \ldots, 2 k_{u}\right\}$, if $f\left(P_{l}\right) \neq P_{l}$ for some $l \in\{1,2, \ldots, p\}$, and $f\left(B_{j}\right) \neq B_{j}$ for some $j \in\{1,2, \ldots, b\}$; 
where $n_{1}, n_{2}, \ldots, n_{s}, r_{i, q}$ for $i=1,2, \ldots, v_{q}$ and $q=1,2, \ldots, \rho$, and $k_{1}, k_{2}$, $\ldots, k_{u}$ are positive integers (non necessarily different) satisfying

$$
1<n_{1}+n_{2}+\ldots+n_{s}+\sum_{q=1}^{\rho} \sum_{i=1}^{v_{q}} \eta_{q} r_{i, q}=b,
$$

$r_{1, q}+r_{2, q}+\ldots+r_{v_{q}, q}=t_{q}$ and $1<k_{1}+k_{2}+\ldots+k_{u}=p$, and $A_{i, q}$ is one of the sets of statements $(a)$ and $(b)$ of Theorem 6 , for all $i=1,2, \ldots, v_{q}$ and $q=1, \ldots, \rho$.

Theorems 4, 5, 6 and 7 are proved in section 4 .

\section{Homology of A GRAPH AND Fuller's RESUlT}

We can consider the fundamental group of a graph $G$, see for instance [10] for more details on the fundamental group. The elements of the fundamental group are oriented loops of $G$. We assume that the fundamental group of $G$ has $c$ independent oriented loops $\gamma_{i}$ for $i=1, \ldots, c$, and let $f: G \rightarrow G$ be a continuous map. Then, the homology groups of $G$ are $H_{0}(X, \mathbb{Q})=\mathbb{Q}$ and $H_{1}(X, \mathbb{Q})=\bigoplus_{i=1}^{c} \mathbb{Q}$, and the actions $f_{* k}: H_{k}(X, \mathbb{Q}) \rightarrow H_{k}(X, \mathbb{Q})$ for $k=0,1$ induced by $f$ on these homology groups are $f_{* 0}=(1)$ (because $G$ is connected) and $f_{* 1}=A$, where $A$ is a $c \times c$ matrix with integer entries. The element $a_{i j}$ of the matrix $A$ is the number of times that the loop $\gamma_{i}$ covers the loop $\gamma_{j}$ taking into account the orientation of the covering. Therefore, the Betti's numbers of $G$ are $B_{0}(G)=\operatorname{dim}_{\mathbb{Q}} H_{0}(X, \mathbb{Q})=1$ and $B_{1}(G)=$ $\operatorname{dim}_{\mathbb{Q}} H_{1}(X, \mathbb{Q})=c$. For more details of the homology of $G$ see $[10,8,11]$.

Fuller in [3] proved the following result; see also Halpern [4] and Brown [2].

Theorem 8 (Fuller's Theorem). Let $f$ be a homeomorphism of a compact polyhedron $X$ into itself. If the Euler characteristic of $X$ is not zero, then $f$ has a periodic point with period not greater than the maximum of $\sum_{k \text { odd }} B_{k}(X)$ and $\sum_{k \text { even }} B_{k}(X)$, where $B_{k}(X)$ denotes the $k$-th Betti number of $X$.

Applying the Fuller's theorem to a graph $G$ it follows Theorem 1.

For other results on the set of a continuous map from a graph into itself see for instance [1] or [6], and the references quoted there.

\section{An interval AND A CIRCLE}

First we state a general result for the set of periods of a homeomorphism of a graph.

Proposition 9. Let $f: G \rightarrow G$ be a homeomorphism of a graph $G$ non homeomorphic to a circle. Then, the following statements hold.

(a) Let $z$ be a vertex of valence $k$. Then $f(z)$ is a vertex of valence $k$ if $k \neq 2$. 
(b) $\operatorname{Per}(f) \neq \emptyset$.

Proof. Statement (a) follows immediately from the definition of a homeomorphism. Since a graph non-homeomorphic to a circle has a vertex with valence different from 2, statement (b) follows easily from statement (a) because a graph has finitely many vertices.

From now on we shall investigate the possible sets $\operatorname{Per}(f)$ for the homeomorphisms $f: G \rightarrow G$ of different graphs $G$. We shall start with the easiest graphs, as an interval and a circle, and we shall finish with more complicated graphs. The results on the set of periods for the homeomorphisms of an interval and of a circle play a main role in the study of the set of periods of the homeomorphisms of other graphs.

Proof of Theorem 2 (Interval Theorem). Without loss of generality, we can suppose that $I=[0,1]$. Hence, if $f:[0,1] \rightarrow[0,1]$ is an orientation preserving homeomorphism (i.e. monotone increasing), by Proposition 9(a) we have $f(0)=0$ and $f(1)=1$. Moreover, we claim that any orbit of $f$, i.e. for all $x \in[0,1]$ we have that $\left\{x, f(x), f^{2}(x), \ldots\right\}$, tends to a fixed point.

Firstly, we remark that if $f:[0,1] \rightarrow[0,1]$ is an increasing homeomorphism, then besides the fixed points 0 and 1, there can exist other fixed points into the interval $I=[0,1]$. We restrict $f$ to a subinterval formed by two consecutive fixed points, i.e. $[y, z]$ such that either $f(x)>$ $x$ for all $x \in(y, z)$ or $f(x)<x$ for all $x \in(y, z)$. If $f(x)>x$ then any orbit $\left\{x, f(x), f^{2}(x), \ldots\right\}$ tends to the fixed point $z$, and if $f(x)<x$ then any orbit $\left\{x, f(x), f^{2}(x), \ldots\right\}$ tends to the fixed point $y$. More precisely, we take $x \in(y, z)$ and first we consider the case that $f(x)>x$. Then, $f^{2}(x)=f(f(x))>f(x)$, because $\left.f\right|_{(y, z)}$ is monotone increasing. By induction we get $f^{n}(x)=f\left(f^{n-1}(x)\right)>f^{n-1}(x)$. Hence, the sequence $\left\{f^{n}(x)\right\}_{n=0}^{\infty}$ is monotone increasing and upper bounded by $z$, so it converges to $\sup \left\{f^{n}(x)\right.$, for $\left.n=0,1, \ldots\right\}=z$. Therefore, the $\omega$-limit set of the orbit of $x \in(y, z)$ is the fixed point $z$.

Similarly, if $f(x)<x$, then the sequence $\left\{f^{n}(x)\right\}_{n=0}^{\infty}$ is decreasing and lower bounded by $y$, and it converges to the fixed point $y$ of $f$. Hence, the claim is proved.

If $f:[0,1] \rightarrow[0,1]$ is an orientation reversing homeomorphism (i.e. monotone decreasing), then by Proposition 9(a) we get $f(0)=1$ and $f(1)=0$. So, $2 \in \operatorname{Per}(f)$. By the Bolzano's Theorem also called the Intermediate Value Theorem, we get that $1 \in \operatorname{Per}(f)$. On the other hand, the second iterate $f^{2}$ is an orientation preserving homeomorphism, so from the first part we obtain $\operatorname{Per}\left(f^{2}\right)=\{1\}$. Therefore, $\operatorname{Per}(f)=\{1,2\}$.

For studying the set of periods of the homeomorphisms of the circle we need to introduce an important dynamical invariant called the rotation number, it was firstly introduced by Poincaré [9] in 1885. 
For studying the dynamics of a continuous map $f: \mathbb{S}^{1} \rightarrow \mathbb{S}^{1}$ it is helpful to lift the map to the straight line $\mathbb{R}$. For a such $f$ we call a map $F: \mathbb{R} \rightarrow \mathbb{R}$ a lifting of $f$ if $\pi \circ F=f \circ \pi$, where $\pi: \mathbb{R} \rightarrow \mathbb{S}^{1}$ is given by $\pi(x)=\exp (2 \pi i x)=$ $\cos (2 \pi x)+\sin (2 \pi x) i$. The degree of the map $f$ is by definition the integer $F(1)-F(0)$, for more details see [1].

There are always infinitely many different liftings for a continuous map $f: \mathbb{S}^{1} \rightarrow \mathbb{S}^{1}$. Indeed, one may easily prove that any two liftings of $f$ differ by an integer, that is, if $F_{1}$ and $F_{2}$ are liftings, then there exists $k \in \mathbb{Z}$ such that $F_{1}(x)=F_{2}(x)+k$.

Let $f: \mathbb{S}^{1} \rightarrow \mathbb{S}^{1}$ be a homeomorphism. If $f$ is orientation preserving, then its degree is 1 and, if $f$ is orientation reversing, its degree is -1 . Moreover, the lifting of a homeomorphism of the circle is a homeomorphism on the straight line.

For studying the set of periods of the orientation preserving homeomorphisms we introduce the rotation number, which is a number between 0 and 1 that roughly speaking measures the average amount of points which are rotated by an iteration of a continuous map $f: \mathbb{S}^{1} \rightarrow \mathbb{S}^{1}$ of degree 1 . Before defining the rotation number, we introduce a preliminary concept.

Let $F$ be a lifting of an orientation-preserving homeomorphism $f: \mathbb{S}^{1} \rightarrow$ $\mathbb{S}^{1}$ of degree 1 . For $x \in \mathbb{S}^{1}$ we define

$$
\rho_{0}(F, x)=\lim _{n \rightarrow \infty} \frac{F^{n}(x)}{n} .
$$

This limit exists and does not depend upon the choice of $x$. For this reason we can put $\rho_{0}(F)$ instead of $\rho_{0}(F, x)$. The rotation number of $f, \rho(f)$, is the fractional part of $\rho_{0}(F)$ for any lifting $F$ of $f$. That is, $\rho(f)$ is the unique number in $[0,1)$ such that $\rho_{0}(F)-\rho(f)$ is an integer. For more details about the rotation number see $[7,5,1]$. We note that in [1] the rotation number is essentially defined as $\rho_{0}(F)$, instead of its fractional part.

Proof of Theorem 3 (Circle Theorem). Let $f: \mathbb{S}^{1} \rightarrow \mathbb{S}^{1}$ be a homeomorphism and assume that it preserves the orientation. Poincaré [9] proved that the rotation number of an orientation preserving homeomorphism is irrational if and only if it has no periodic points, see also [1]. So, for proving statement (a) we only need to prove the equality $\operatorname{Per}(f)=\{n\}$ when $\rho(f)=k / n$ with $\operatorname{gcd}(k, n)=1$, and this is proved for instance in [5, 1]. So, the proof of statement (a) is completed.

Suppose that $f$ reverses the orientation. Since continuous maps of degree -1 have fixed points, see for instance [1], we have that $1 \in \operatorname{Per}(f)$. So, there exists a point $x \in \mathbb{S}^{1}$ such that $f(x)=x$. Then $f^{2}(x)=x$ and, as $f^{2}$ is a homeomorphism that preserves the orientation, by statement (a) we get $\operatorname{Per}\left(f^{2}\right)=\{1\}$ and, consequently, $\operatorname{Per}(f) \subseteq\{1,2\}$.

If we consider now the circle as the interval $[0,1]$ with both endpoints identified, the map $f:[0,1] \rightarrow[0,1]$ defined by $f(x)=1-x$ is such that $f^{2}$ is the identity. So, for this orientation reversing homeomorphism we have that 
$\operatorname{Per}(f)=\{1,2\}$. Now, there are monotone decreasing maps $g:[0,1] \rightarrow[0,1]$ such that $g(0)=1, g(1)=0$ and $\operatorname{Per}(g)=\{1\}$. For example, consider a decreasing map $g:[0,1] \rightarrow[0,1]$ such that $g(0)=1, g(1)=0, g\left(x_{0}\right)=x_{0}>$ $1 / 2, g(x)>\frac{x_{0}-1}{x_{0}} \cdot x+1$ for all $x \in\left[0, x_{0}\right]$ and $g(x)=\frac{x_{0}}{x_{0}-1} \cdot(x-1)$ for all $x \in\left[x_{0}, 1\right]$, where $x_{0}$ is a fixed point of $g$ into the interval $\left(\frac{1}{2}, 1\right)$. From the definition of $g$ we get that $g(x)>1-x$ for all $x \in(0,1)$ and that these maps are orientation reversing homeomorphism such that $\operatorname{Per}(f)=\{1\}$. This completes the proof of statement (b).

\section{A $p$-FLOWER GRAPH, A $b$-ODD GRAPH, AN $n$-LIPS GRAPH AND A $(p, b)$-GRAPH}

In these section we shall prove Theorems 4, 5, 6 and 7 .

Proof of Theorem 4 ( $p$-Flower Theorem). Let $G$ be a $p$-flower graph with the branching point $z$ and $p$ petals $P_{1}, P_{2}, \ldots, P_{p}$. If $f: G \rightarrow G$ is a homeomorphism, by Proposition 9(a) we have that $f(z)=z$. Then, $1 \in \operatorname{Per}(f)$.

Assume that $f\left(P_{l}\right)=P_{l}$ for all $l=1,2, \ldots, p$. Then, $\left.f\right|_{P_{l}}: P_{l} \rightarrow P_{l}$ is a homeomorphism of the topological circle $P_{l}$ with a fixed point $z$. So, from Theorem 3 it follows that $\operatorname{Per}(f)=\{1\}$ or $\operatorname{Per}(f)=\{1,2\}$, and statement (a) is proved.

Suppose that $f\left(P_{l}\right) \neq P_{l}$ for some $l \in\{1,2, \ldots, p\}$. Since every petal must be applied to another petal by $f$, there exist $n_{1}$ petals $P_{k_{1}}, P_{k_{2}}, \ldots, P_{k_{n_{1}}}$ such that $f\left(P_{k_{i}}\right)=P_{k_{i+1}}$, for all $i=1,2, \ldots, n_{1}-1$, and $f\left(P_{k_{n_{1}}}\right)=P_{k_{1}}$, where $1<n_{1} \leq p$. Therefore, the iterate $f^{n_{1}}$ is a homeomorphism of the topological circle $P_{k_{1}}$ having a fixed point. Thus, $\operatorname{Per}\left(f^{n_{1}}\right)$ is $\{1\}$ or $\{1,2\}$. Therefore, either $1 \in \operatorname{Per}(f)$, or $\left\{1, n_{1}\right\} \subset \operatorname{Per}(f)$, or $\left\{1,2 n_{1}\right\} \subset \operatorname{Per}(f)$, or $\left\{1, n_{1}, 2 n_{1}\right\} \subset \operatorname{Per}(f)$.

Furthermore, if $n_{1}<p$, there can exist other $n_{2}$ petals $P_{l_{1}}, P_{l_{2}}, \ldots, P_{l_{n_{2}}}$ with similar property and satisfying $1 \leq n_{2} \leq p-n_{1}$, implying that either $1 \in \operatorname{Per}(f)$, or $\left\{1, n_{2}\right\} \subset \operatorname{Per}(f)$, or $\left\{1,2 n_{2}\right\} \subset \operatorname{Per}(f)$, or $\left\{1, n_{2}, 2 n_{2}\right\} \subset$ $\operatorname{Per}(f)$.

In short, repeating these arguments, there can exist $n_{1}, n_{2}, \ldots, n_{s}$ positive integers with the above properties such that either $1 \in \operatorname{Per}(f)$, or $\left\{1, n_{i}\right\} \subset$ $\operatorname{Per}(f)$, or $\left\{1,2 n_{i}\right\} \subset \operatorname{Per}(f)$, or $\left\{1, n_{i}, 2 n_{i}\right\} \subset \operatorname{Per}(f)$, for all $i=1,2, \ldots, s$, and satisfying $1<n_{1}+n_{2}+\ldots+n_{s}=p$. Reordering the numbers $n_{i}$ if necessary, statement (b) follows.

Proof of Theorem 5 ( $b$-odd Theorem). Let $G$ be a $b$-odd graph with branching point $z$ and edges $B_{1}, B_{2}, \ldots, B_{b}$. If $f: G \rightarrow G$ is a homeomorphism, by Proposition 9(a) we have that $f(z)=z$. Then, $1 \in \operatorname{Per}(f)$.

If $f$ fixes the other vertices, that is, $f(x)=x$ for all $x \in \mathrm{V}(G)$, from Theorem 2, we get that $\operatorname{Per}(f)=\{1\}$. Otherwise, since the image of an edge by the homeomorphism $f$ is another edge, there exist $n_{1}$ edges $B_{k_{1}}, B_{k_{2}}, \ldots, B_{k_{n_{1}}}$ such that $f\left(B_{k_{i}}\right)=B_{k_{i+1}}$, for all $i=1,2, \ldots, n_{1}-1$, and 
$f\left(B_{k_{n_{1}}}\right)=B_{k_{1}}$, where $1<n_{1} \leq b$. Therefore, the iterate $f^{n_{1}}$ is a homeomorphism of the topological interval $B_{k_{1}}$ having two fixed points, that is, the branching point $z$ and the other vertex of $B_{k_{1}}$. Thus, $\operatorname{Per}\left(f^{n_{1}}\right)=\{1\}$. Hence, $\left\{1, n_{1}\right\} \subset \operatorname{Per}(f)$ because the vertices of $B_{k_{i}}$ different from $z$ form a periodic orbit of period $n_{1}$.

Furthermore, if $n_{1}<b$, there exist other $n_{2}$ edges $B_{l_{1}}, B_{l_{2}}, \ldots, B_{l_{n_{2}}}$ with similar property satisfying $1 \leq n_{2} \leq b-n_{1}$, implying that $n_{2} \in \operatorname{Per}(f)$.

In short, repeating these arguments there can exist $n_{1}, n_{2}, \ldots, n_{s}$ positive integers with the above properties such that $n_{i} \in \operatorname{Per}(f)$, for all $i=1,2, \ldots, s$, and satisfying $1<n_{1}+n_{2}+\ldots+n_{s}=b$. Reordering the numbers $n_{i}$ if necessary, it follows the result.

Proof of Theorem 6 ( $n$-lips Theorem). Let $G$ be an $n$-lips graph with vertices $z$ and $w$, and let $e_{i}$ be the edges of $G$ for $i=1,2, \ldots, n$. If $f: G \rightarrow G$ is a homeomorphism and $f(z)=z$, by Proposition 9 (a) we have that $f(w)=w$ and then $1 \in \operatorname{Per}(f)$. But if $f(z) \neq z$ the Proposition 9(a) assures that $f(z)=w$ and $f(w)=z$ and hence $2 \in \operatorname{Per}(f)$.

Assume that $f(z)=z$ and $f\left(e_{i}\right)=e_{i}$ for all $i=1,2, \ldots, n$. Then, for each $i=1,2, \ldots, n,\left.f\right|_{e_{i}}: e_{i} \rightarrow e_{i}$ is an increasing homeomorphism. So, by Theorem 2, follows that $\operatorname{Per}(f)=\{1\}$. So, statement (a) is proved.

Now assume that $f(z)=z$ and $f\left(e_{i}\right) \neq e_{i}$ for some $i \in\{1,2, \ldots, n\}$. Since the image of the edge $e_{i}$ by the homeomorphism $f$ is another edge, there exist $n_{1}$ edges $e_{k_{1}}, e_{k_{2}}, \ldots, e_{k_{n_{1}}}$ such that $f\left(e_{k_{i}}\right)=e_{k_{i+1}}$, for all $i=1,2, \ldots, n_{1}-1$, and $f\left(e_{k_{n_{1}}}\right)=e_{1}$, where $1<n_{1} \leq n$. Therefore, the iterate $f^{n_{1}}$ is an increasing homeomorphism of the topological interval $e_{k_{1}}$ having a fixed point. Thus, by Theorem $2 \operatorname{Per}\left(\left.f^{n_{1}}\right|_{e_{k_{1}}}\right)=\{1\}$. Hence, $\operatorname{Per}(f)$ contains either $\{1\}$ or $\left\{1, n_{1}\right\}$.

Furthermore, if $n_{1}<n$, there exist other $n_{2}$ edges $e_{l_{1}}, e_{l_{2}}, \ldots, e_{l_{n_{2}}}$ with similar property satisfying $1 \leq n_{2} \leq n-n_{1}$, implying that $\operatorname{Per}(f)$ contains either $\{1\}$, or $\left\{1, n_{1}\right\}$, or $\left\{1, n_{2}\right\}$, or $\left\{1, n_{1}, n_{2}\right\}$.

In short repeating these arguments there can exist $n_{1}, n_{2}, \ldots, n_{s}$ nonnegative integers with the above properties such that $1 \in \operatorname{Per}(f)$ and eventually $n_{i} \in \operatorname{Per}(f)$, for all $i=1,2, \ldots, s$, and satisfying $1<n_{1}+n_{2}+\ldots+n_{s}=$ $n$. Reordering the numbers $n_{i}$ if necessary, statement (b) follows.

Suppose that $f(z) \neq z$ and $f\left(e_{i}\right)=e_{i}$ for all $i=1,2, \ldots, n$. Then, for each $i=1,2, \ldots, n,\left.f\right|_{e_{i}}: e_{i} \rightarrow e_{i}$ is a decreasing homeomorphism. So, by Theorem 2, follows that $\operatorname{Per}(f)=\{1,2\}$. So, statement (c) is proved.

In the case that $f(z) \neq z$ and $f\left(e_{i}\right) \neq e_{i}$ for some $i=1,2, \ldots, n$ we use the same argument that in statement (b) and we obtain a positive integer $n_{1}$ such that the iterate $f^{n_{1}}$ is a homeomorphism of some topological interval $e_{m}$, where $m \in\{1,2, \ldots, n\}$. But here, we note that if $n_{1}$ is odd, then $f^{n_{1}}$ is a decreasing homeomorphism implying that $\operatorname{Per}(f)$ contains either $\left\{2, n_{1}\right\}$ or 
$\left\{2, n_{1}, 2 n_{1}\right\}$. And if $n_{1}$ is even, then $f^{n_{1}}$ is an increasing homeomorphism implying that either $2 \in \operatorname{Per}(f)$ or $\left\{2, n_{1}\right\} \subset \operatorname{Per}(f)$. Hence, repeating the argument used in statement (b) we conclude that there can exist $n_{1}, n_{2}, \ldots, n_{s}$ non-negative integers such that either $n_{i} \in \operatorname{Per}(f)$, or $\left\{n_{i}, 2 n_{i}\right\} \subset \operatorname{Per}(f)$ for all $i=1,2, \ldots, s$, and satisfying $1<n_{1}+n_{2}+\ldots+n_{s}=n$. Of course, the case $\left\{n_{i}, 2 n_{i}\right\} \subset \operatorname{Per}(f)$ only can occur if $n_{i}$ is odd. So, statement (d) follows.

Consider a branching point $z$ with valence $k$. This valence can be decomposed as $k=2 p+b$, where $p+b>0, p \geq 0$ is the number of all petals with endpoint $z$ and $b \geq 0$ is the number of edges which are not petals with endpoint $z$. In this case we shall say that the branching point $z$ is of type $(p, b)$. Then every vertex of a graph has a type $(p, b)$. For example, an endpoint is a vertex of valence $k=1$, and hence, it is of the type $(0,1)$. Now we can improve Proposition 9(a) as follows.

Proposition 10. Let $f: G \rightarrow G$ be a homeomorphism of a graph $G$ non homeomorphic to a circle. If $z$ is a vertex of type $(p, b)$, then $f(z)$ is a vertex of type $(p, b)$.

Proof. It follows immediately from the definition of a homeomorphism.

Proof of Theorem $7((p, b)$-graph Theorem $)$. Let $G$ be a $(p, b)$-graph with the main branching point $z, p$ petals $P_{1}, P_{2}, \ldots, P_{p}$, and $b$ edges $B_{1}, B_{2} \ldots, B_{b}$ which are not petals. Assume that $L_{j_{q}, 1}^{\eta_{q}}, L_{j_{q}, 2}^{\eta_{q}}, \ldots, L_{j_{q}, t_{q}}^{\eta_{q}}$ are the $\eta_{q}$-lips for $q=1,2, \ldots, \rho$ contained into the $(p, b)$-graph described in the statement of the theorem. If $f: G \rightarrow G$ is a homeomorphism, by Proposition 10 we have that $f(z)=z$. Then $1 \in \operatorname{Per}(f)$.

Assume that $f\left(P_{l}\right)=P_{l}$ for all $l=1,2, \ldots, p$, and $f\left(B_{j}\right)=B_{j}$ for all $j=$ $1,2, \ldots, b$. Then, for each $l=1,2, \ldots, p,\left.f\right|_{P_{l}}: P_{l} \rightarrow P_{l}$ is a homeomorphism of the topological circle $P_{l}$ with the fixed point $z$, and, for each $j=1,2, \ldots, b$, $\left.f\right|_{B_{j}}: B_{j} \rightarrow B_{j}$ is a homeomorphism of the topological interval $B_{j}$ with the fixed endpoint $z$. So, from Theorems 2 and 3 it follows that either $\operatorname{Per}(f)=\{1\}$, or $\operatorname{Per}(f)=\{1,2\}$, and statement (a) is proved.

Suppose that $f\left(P_{l}\right)=P_{l}$ for all $l=1,2, \ldots, p$, and $f\left(B_{j}\right) \neq B_{j}$ for some $j \in\{1,2, \ldots, b\}$. For the $p$ petals we apply Theorem 3 and we obtain that either $\{1\} \subset \operatorname{Per}(f)$, or $\{1,2\} \subset \operatorname{Per}(f)$. Since every edge which is not a petal must be applied to another edge which is not a petal by $f$, we apply Theorem 5 to the $n$-odd subgraph of $G$ formed by all the edges which are not petals and are not contained into the $\eta_{q}$-lips $L_{j_{q}, k}^{\eta_{q}}$, for $q=1,2, \ldots, \rho$ and $k=1,2, \ldots, t_{q}$. We conclude that there exist $n_{1}, n_{2}, \ldots, n_{s}$ positive integers such that $n_{i} \in \operatorname{Per}(f)$, for all $i=1,2, \ldots, s$, and satisfying $1<$ $n_{1}+n_{2}+\ldots+n_{s} \leq b$. Furthermore, if $f\left(B_{j}\right) \neq B_{j}$ for some edge $B_{j}$ of some $\eta_{q}$-lips $L_{j_{q}, k}^{\eta_{q}}$, for $q=1,2, \ldots, \rho$ and $k=1,2, \ldots, t_{q}$, since every $\eta_{q}$-lips must be applied into another $\eta_{q}$-lips by $f$ there can exist $r_{1, q} \leq t_{q}$ $\eta_{q}$-lips' forming a cycle, i.e. there are $L_{j_{q}, m_{1}}^{\eta_{q}}, L_{j_{q}, m_{2}}^{\eta_{q}}, \ldots, L_{j_{q}, m_{r_{1, q}}}^{\eta_{q}}$ such that 
$f\left(L_{j_{q}, m_{i}}^{\eta_{q}}\right)=L_{j_{q}, m_{i+1}}^{\eta_{q}}$ for all $i=1,2, \ldots, r_{1, q}-1$ and $f\left(L_{j_{q}, m_{r_{1, q}}}^{\eta_{q}}\right)=L_{j_{q}, m_{1}}^{\eta_{q}}$. Thus, the iterate $f^{r_{1, q}}$ is a homeomorphism from the $\eta_{q}$-lips $L_{j_{q}, m_{1}}^{\eta_{q}}$ into itself. Since the branching point $z$ is fixed by $f$ we get that $\operatorname{Per}\left(\left.f^{r_{1, q}}\right|_{L_{j_{q}, m_{1}}^{\eta_{q}}}\right)$ is a set $A_{1, q}$ as one of the sets of statements (a) and (b) of Theorem 6 . Therefore we get that the set $r_{1, q} A_{1, q} \subset \operatorname{Per}(f)$.

Furthermore, if $r_{1, q}<t_{q}$ there exist others $r_{2, q} \eta_{q}$-lips' $L_{j_{q}, a_{1}}^{\eta_{q}}, L_{j_{q}, a_{2}}^{\eta_{q}}, \ldots$, $L_{j_{q}, a_{r_{2, q}}}^{\eta_{q}}$ with similar property satisfying $1 \leq r_{1, q}+r_{2, q} \leq t_{q}$, implying that $\operatorname{Per}\left(\left.f^{r_{2, q}}\right|_{L_{j q, a_{1}}^{\eta_{q}}}\right)$ is a set $A_{2, q}$ as one of the sets of statements (a) or (b) of Theorem 6. Therefore we have that $r_{2, q} A_{2, q} \subset \operatorname{Per}(f)$.

In short, repeating these arguments there can exist $r_{1, q}, r_{2, q}, \ldots, r_{v_{q}, q}$ positive integers and $A_{1, q}, A_{2, q}, \ldots, A_{v_{q}, q}$ sets being as one of the sets of statements (a) or (b) of Theorem 6 such that

$$
\bigcup_{q=1}^{\rho}\left(\bigcup_{i=1}^{v_{q}} r_{i, q} A_{i, q}\right) \subset \operatorname{Per}(f),
$$

with $r_{1, q}+r_{2, q}+\ldots+r_{v_{q}, q}=t_{q}$ and

$$
n_{1}+n_{2}+\ldots+n_{s}+\sum_{q=1}^{\rho} \sum_{i=1}^{v_{q}} \eta_{q} r_{i, q}=b .
$$

Reordering the numbers $n_{j}$ and $r_{i, q}$ if necessary, statement (b) follows.

When $f\left(P_{l}\right) \neq P_{l}$ for some $l \in\{1,2, \ldots, p\}$, and $f\left(B_{j}\right)=B_{j}$ for all $j=1,2, \ldots, b$, by applying Theorem 5 to the $b$ edges which are not petals we get that $1 \in \operatorname{Per}(f)$. Then, by using the fact that every petal must be applied to another petal by $f$, we apply statement (b) of Theorem 4 to the $p$ petals and we obtain statement (c).

In the case that $f\left(P_{l}\right) \neq P_{l}$ for some $l \in\{1,2, \ldots, p\}$, and $f\left(B_{j}\right) \neq B_{j}$ for some $j \in\{1,2, \ldots, b\}$, we apply statement (b) of Theorem 4 to the $p$ petals, and Theorems 5 and statements (a) and (b) of Theorem 6 to the other $b$ edges which are not petals, using the same arguments than in the proof of statements (b) and (c) we get statement (d).

\section{ACKNOWLEDGMENTS}

The first author is partially supported by CAPES/DGU grant number BEX 12566/12-8. The second author is partially supported by a MINECO/ FEDER grant number MTM2008-03437, by a AGAUR grant number 2009 SGR 410, by ICREA Academia and by FP7-PEOPLE-2012-IRSES 316338 and 318999. Both authors are partially supported by the project PHB 20090025-PC. 


\section{REFERENCES}

[1] L. Alsedá, J. Llibre and M. Misiurewicz, Combinatorial Dynamics and Entropy in Dimension One, Second Edition, World Scientific, Singapore, 2001.

[2] R.F. Brown, The Lefschetz fixed point theorem, Scott, Foresman and Company, Glenview, IL, 1971.

[3] F. B. Fuller, The existence of periodic points, Ann. of Math. 57 (1953), 229-230.

[4] B. Halpern, Fixed points for iterates, Pacific J. of Math. 25 (1968), 255-275.

[5] A. Katok and B. Hasselblatt, Introduction to the modern theory of dynamical systems, Cambridge University Press, 1995.

[6] J. Llibre, Periodic point free continuous self-maps on graphs and surfaces, Topology and its Applications 159 (2012), 2228-2231.

[7] W. de Melo and S. van Strien, One-dimensional Dynamics, Springer, Berlin, 1993.

[8] J. R. Munkres, Elements of Algebraic Topology, Addison-Wesley, 1984.

[9] H. Poincaré, Sur les courbes définies par les équations differéntielles, J. de Mathématiques, série 4, 1 (1885), 167-244.

[10] E.H. Spanier, Algebraic Topology, McGraw-Hill Book Company, New York, 1966.

[11] J. W. Vicks, Homology Theory: An Introduction to Algebraic Topology, SpringerVerlag, New York, 1994; Academic Press, New York, 1973.

${ }^{1}$ Departament de Matemàtiques, Universitat Autònoma de Barcelona, 08193 Bellaterra, Barcelona, Catalonia, Spain

E-mail address: jllibre@mat.uab.cat

2 Departamento de Matemática, ibilce-Unesp, Rua C. Colombo, 2265, CEP 15054-000 S. J. Rio Preto, SÃo Paulo, Brazil

E-mail address: maralves@ibilce.unesp.br 